\title{
Surgery of Anterior Communicating Artery Aneurysms
}

\author{
Yoko Kato, Mohsen Nouri, and Guowei Shu
}

\subsection{Sign and Symptoms}

Anterior communicating artery (AComA) aneurysms are among the most common aneurysms in different case series. They compose 36 of 175 cases $(20.6 \%)$ in our latest case series of unruptured aneurysms coming second only after middle cerebral artery aneurysms (69 cases) [1]. In case series of ruptured aneurysms, the incidence is higher, and around $30-40 \%$ of all intracranial aneurysms are located in the AcomA region. Their presentation with subarachnoid hemorrhage (SAH) is not essentially different from other aneurysms and will not be repeated in this chapter again. Here, we do not intend to make an exhaustive review of different aspects of medical, interventional, or surgical management of these aneurysms. Instead, we will explain the most important pre-, intra-, and postoperative aspects observed in our institute.

\footnotetext{
Y. Kato $(\bowtie)$

Department of Neurosurgery, Fujita Health University, Toyoake, Aichi, Japan e-mail: kyoko@fujita-hu.ac.jp

\section{Nouri}

Gundishapour Academy of Neuroscience, Ahvaz, Iran e-mail: nouri@gan-ac.ir

\section{G. Shu}

Department of Neurosurgery, Shuguang Hospital, Shanghai University of Traditional Chinese Medicine, Shanghai, China
}

\subsection{Investigation}

To evaluate any patient with subarachnoid hemorrhage or any candidate for aneurysm screening, we request 3D CT angiography (CTA), and digital subtraction angiography is performed only when 3D-CTA is negative for any aneurysm in spite of high clinical suspicion for one [2].

There are important anatomical points which should be paid attention on CTA that may affect our decision-making before or during the operation. Aneurysm projection, the location of neck and dome of aneurysm, its height and distance from the planum sphenoidale, and most condition of A2 fork are the factors affecting our surgical planning. Here, we describe the most important factors and explain how they are pivotal for decision-making.

\subsubsection{Aneurysm Projections}

Figure 14.1 demonstrates four types of projections of AComA aneurysms.

Each of these projections may obscure some portions of the contralateral A1, A2, or optic nerve which is dependent on the side of the approach.

Posterior projecting aneurysms require special attention: very important tiny perforators are stretched over the posterior wall of these aneu- 
rysms. When approached from the side with A2 posterior to the aneurysm, the neck or the posterior stretched perforating arteries cannot be reached and dissected efficiently.

\subsubsection{A2 Fork}

Bilateral A2s and the AComA are named A2 fork. At the level of AComA, one A2 is usually anterior to the other making the AComA run obliquely instead of in the coronal plane.

a
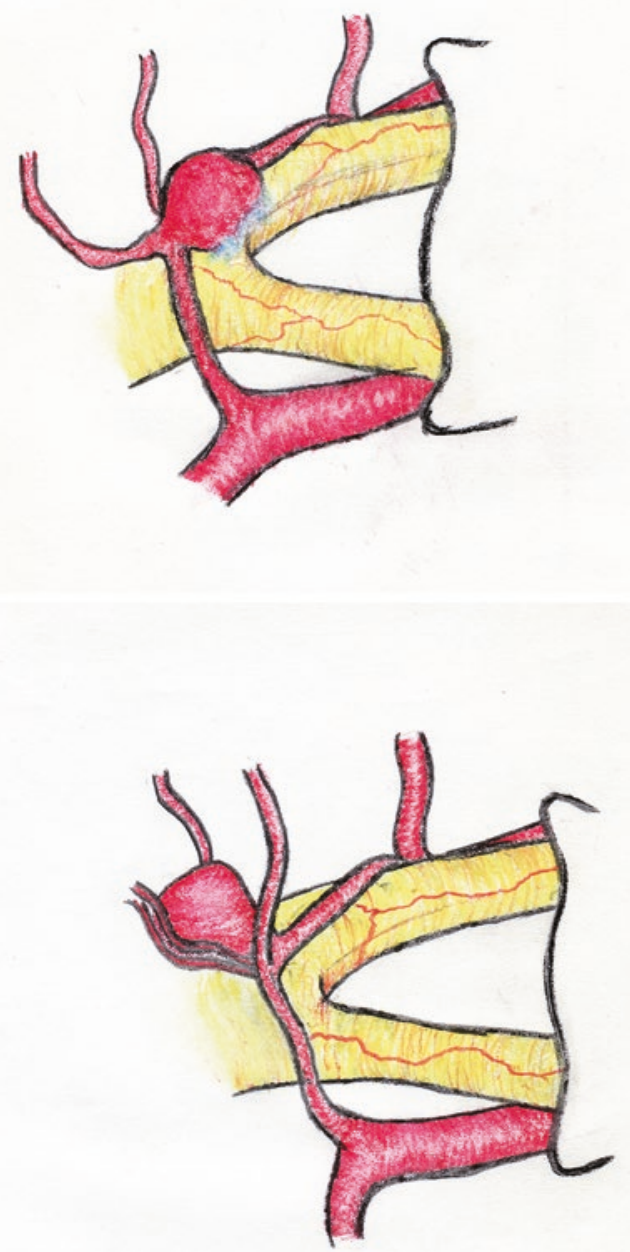

Fig. 14.1 Different projections of anterior communicating artery aneurysms. (a) Inferior-, (b) anterior-, (c) posterior-, and (d) superior-looking aneurysms. All the views are from open A2 fork except for the posterior-looking
When the complex is approached from the side with the anteriorly located A2, the AComA is hidden behind it, and so, the complex is called closed A2 fork. In contrary, when the same complex is approached from the side with the A2 located posteriorly, the AComA is very well visible, and the complex is named open A2 fork (Fig. 14.1).

We approach the AComA aneurysms from the side with open A2 fork because the neck of the aneurysm is usually anterior or superior to the AComA and visible from this side. Posterior-looking aneurysms are exception to this rule, as their neck is

b

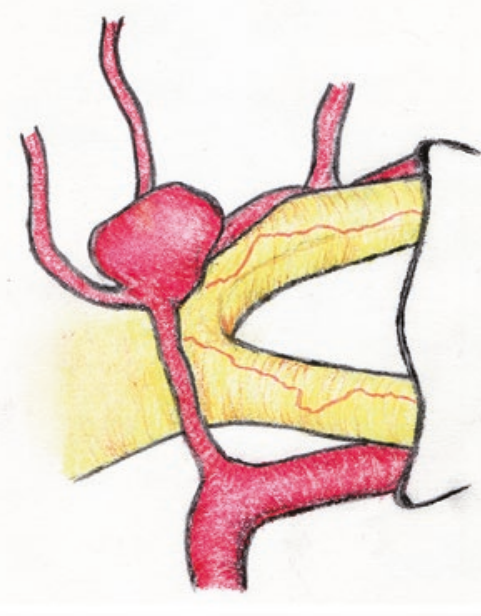

d

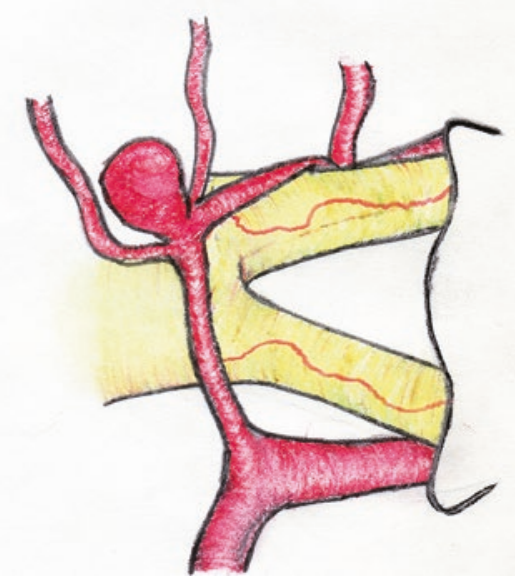

aneurysm (d) which is viewed from the close A2 angle (i.e., the ipsilateral A2 is anterior to the contralateral one). Anterior- and inferior-looking aneurysms (a and b) may conceal the contralateral A1 
located posterior to the AComA and should be approached from the side with the closed fork.

\subsection{Preoperative Preparation}

Although recommended by some authors, inserting a lumbar drain to relax the brain is not necessary in our idea. We administer a dosage of mannitol $(1 \mathrm{~g} / \mathrm{kg})$ just before the incision, and if blood pressure is not too low, we inject furosemide (20-40 mg) as well. Routine precautions for patient positioning (e.g., padding and elastic bandage of the lower limbs) are taken.

We use intraoperative indocyanine green (ICG) video angiography (VA) to check every step of the surgery [3]. If not available, intraoperative conventional angiography is recommended to make sure of appropriate clipping and preservation of the vessels at the conclusion of the surgery. Also, we use intraoperative endoscopy to check anatomical details hidden under microscope before and after clipping [4].

\subsubsection{Approach}

To reach AComA aneurysms, several corridors such as pterional, orbito-zygomatic extension, and interhemispheric approaches have been described. Here, we only explain pterional approach to these lesions as most of the AComA aneurysms can be dealt with this approach. Only for very high located aneurysms, we may use interhemispheric approach. We have not found orbito-zygomatic extension necessary for any of our patients as with proper dissection of the sylvian fissure and cisterns; the aneurysm can be approached without or with only minimal retraction on the frontal lobe.

For pterional transsylvian approach, the patient is positioned supine with his/her trunk elevated about $20^{\circ}$. We use Sugita head holder to secure the patient's head and turn it to the contralateral side between $30^{\circ}$ and $45^{\circ}$ (Fig. 14.2).

Incision is made $5 \mathrm{~mm}$ anterior to the tragus and superior to the zygomatic arc toward the midline (Fig. 14.2). A submuscular or interfascial temporalis flap is turned to expose the bony anatomy.

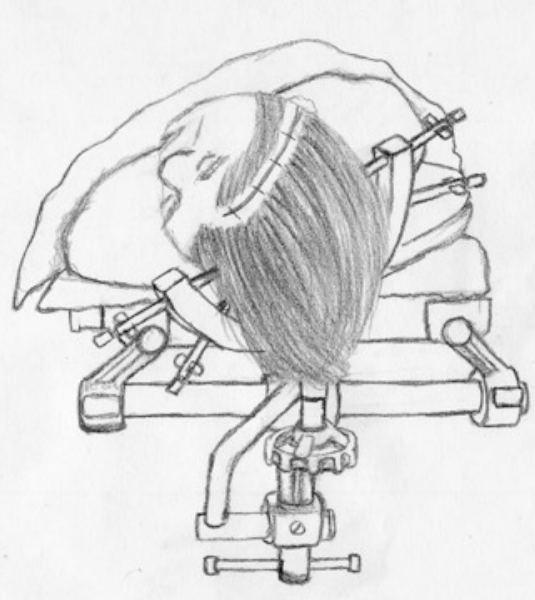

Fig. 14.2 Patient positioning for a classic frontotemporal craniotomy for an anterior communicating artery aneurysm. The incision line is marked on the skin

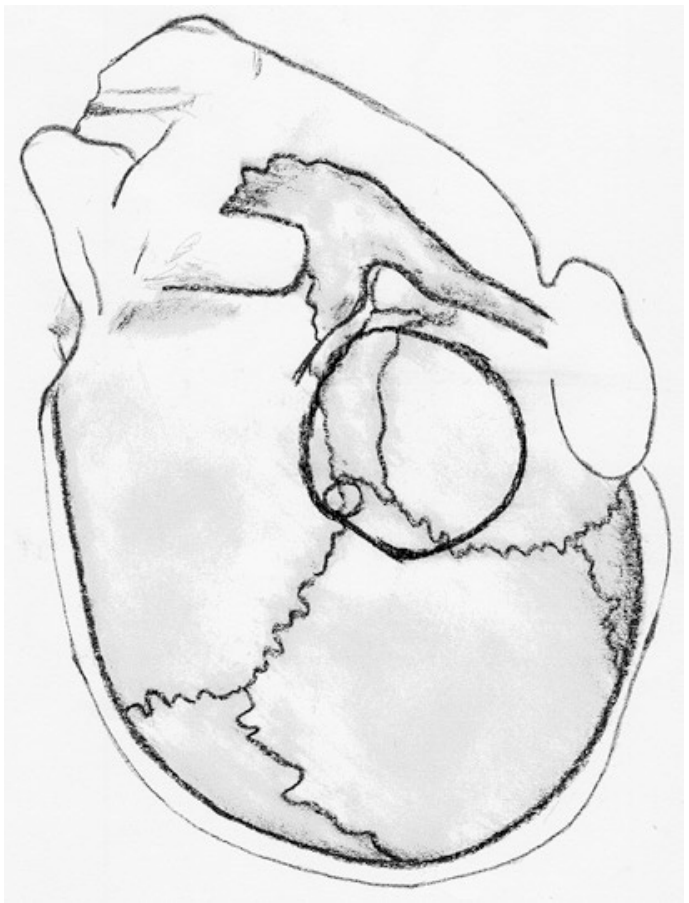

Fig. 14.3 The location of burr hole and craniotomy for a pterional approach for anterior communicating artery aneurysm

One burr hole above the pterion is made, and a fronto-temporo-sphenoidal bone flap is removed with the help of craniotome (Fig. 14.3). The sphenoid ridge is removed with a bone rongeur or high-speed drill. This step helps the surgeon 
to reach the aneurysm with less retraction on the frontal lobe as this bony structure may obscure the field of view. If required, the orbital roof can also be drilled and flattened for a better view of the aneurysm complex.

\subsection{Steps of the Surgery}

The dura is incised with its base toward the pterion (Fig. 14.4). Then, under microscopic magnification, the sylvian fissure is incised with a needle or arachnoid knife. This is important to open the arachnoid layer on the frontal side of the sylvian vein(s) to keep them safe on the temporal side while inserting retraction on the frontal lobe (Fig. 14.5). To have an appropriate view of the aneurysm complex, one should perform a wide sylvian fissure dissection to expose the complex with minimal retraction on the frontal lobe. Surgeon should keep it in mind to release the retractor every now and then to prevent ischemic injury to the frontal lobe.

While opening the sylvian arachnoid layers, a slight retraction on the frontal lobe should be exercised to keep the arachnoid membranes under tension: this makes the dissection with microscissors easier and safer as one can see behind the arachnoid layers. You can use a self-retraining

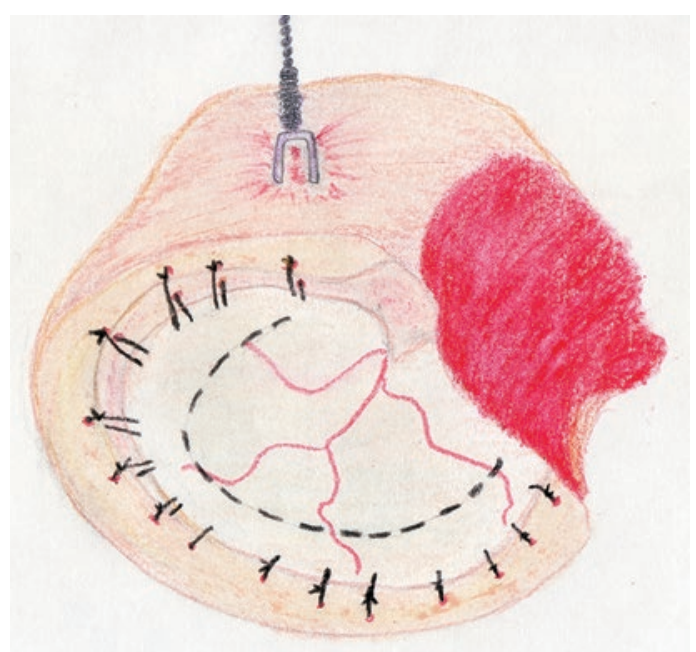

Fig. 14.4 A frontotemporal craniotomy after removing the bone flap. The sphenoid wing and the orbital roof will be flattened with a high-speed drill or bone rongeur. Base of the dura incision is toward the sphenoid wing retractor on the frontal lobe or may use a blunt suction with your non-dominant hand to retract the frontal lobe. The latter requires more experience but is more desirable as less retraction is exerted on the brain parenchyma. Copious or water jet irrigation helps with better sylvian fissure dissection especially in patients with ruptured aneurysms whose arachnoid layers are extremely adhesive. While dissecting the deep sylvian cistern, no artery should be cut, and extreme care should be exercised to preserve all the veins as much as possible. Fine arachnoid trabecula are separated and cut with sharp dissection or occasionally by gentle maneuvers of bipolar tips.

After reaching into the parasellar cisterns, we may require to change the trajectory of the microscope. There is an arachnoid layer connecting the optic nerve to the frontal lobe which we name "white line" or "Sano's line" (Fig. 14.6). This arachnoid is the first to be dissected after opening the sylvian fissure and should be cut $1 \mathrm{~mm}$ away from the frontal lobe to prevent any vascular injury to the brain which may result in cognitive symptoms. Dissecting this line allows the surgeon to mobilize the frontal lobe without retracting the optic complex. By opening the carotid cistern and cerebrospinal fluid (CSF) drainage, we will make the brain even more relax. Arachnoid layer between the ICA and the optic nerve should be dissected until reaching the ICA bifurcation. Dissection of the chiasmatic cistern helps with

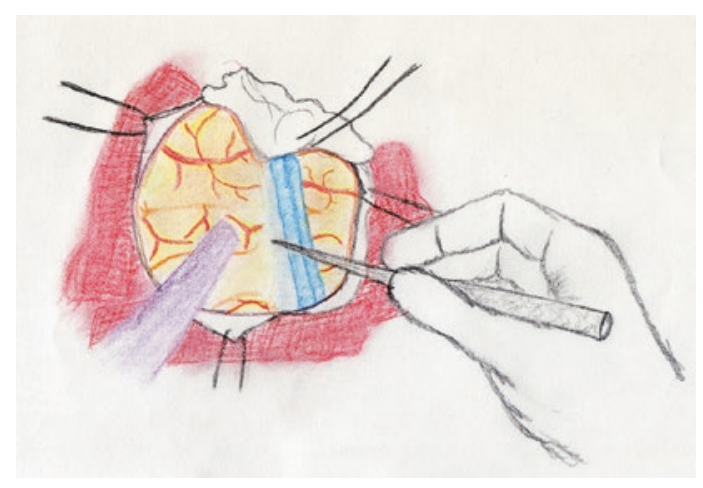

Fig. 14.5 The arachnoid layer between the superficial sylvian vein(s) and the frontal lobe is incised to dissect the sylvian fissure. Slight retraction is put on the frontal lobe to keep the arachnoid layer under constant tension to ease the dissection 


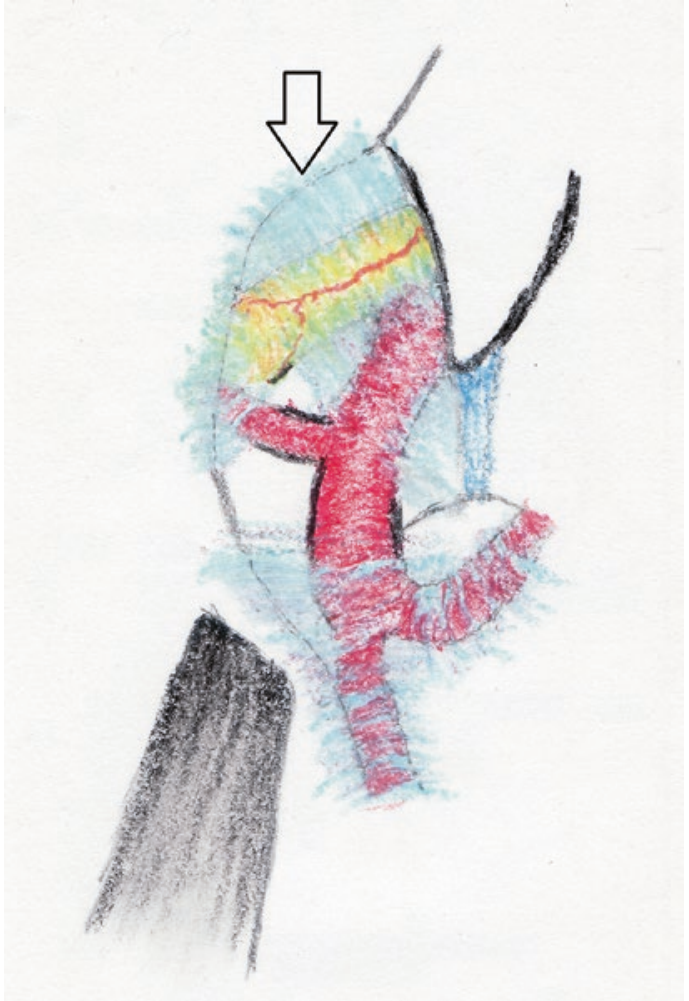

Fig. 14.6 Anterior basal cisterns around the carotid artery, its branches, and the optic nerve. The arrow is referring to the white line or "Sano's line": the arachnoid layer attaching the frontal lobe to the optic nerve

more CSF drainage and easier frontal lobe detachment from the optic apparatus. The cistern of the lamina terminalis should be opened along the inferior border of A1 toward the aneurysm complex. This method preserves the perforating arteries arising from the posterosuperior surface of A1. By following the A1 artery and crossing the optic chiasma, the contralateral A1 is reached and dissected inferiorly to be sure of the contralateral proximal control if needed. However, we do not use proximal temporary clips (either ipsi- or contralaterally) in unruptured aneurysms routinely.

We do not routinely open the lamina terminalis except in hydrocephalic patients where it has been shown to reduce the chance of shunt dependency in the future. When the surgeon decides to open the lamina terminalis, he/she should be careful not to injure optic chiasma inferiorly or anterior commissure superiorly.

\subsubsection{Proximal Control Clipping}

As mentioned above, we do not recommend routine application of proximal control clipping in all cases. In ruptured aneurysms and in large unruptured cases, we advocate proximal clipping to soften the aneurysm and keep on the safe side in case of any premature rupture. The best place for inserting the temporary clip is the middle third of the A1. A more proximally placed clip may injure the perforating arteries arising from the proximal half of A1, and a more distally placed clip may obscure the surgical view and interfere with proper dissection of the aneurysm neck.

\subsubsection{Gyrus Rectus Resection}

Dissecting and separating the interhemispheric fissure to expose an unruptured aneurysm are usually all needed. But for ruptured aneurysms where the interhemispheric fissure is adhesive and for large, high riding or posterior-looking unruptured aneurysms, resection of the adjacent gyrus rectus is recommended. For gyrus rectus resection, place the tip of blade just lateral to the A1 where it disappears in the fissure and medial to the olfactory nerve. Two arteries, namely, recurrent artery of Heubner and fronto-orbital artery should be protected and preserved during the resection.

Set bipolar at minimal possible voltage and coagulate the pia meter of the gyrus rectus. After cutting the pia, start removing the gyrus with a blunt-tipped suction. The medial pia should be left over the aneurysm to avoid inadvertent rupture (Fig. 14.7). Resection of the gyrus is safe as posterior as AComA to avoid the posteriorly located perforating arteries.

\subsubsection{Aneurysm Dissection and Clipping}

Before dissecting the aneurysm, bilateral A1 arteries should be dissected, and with a blunt dissector, check for the probable place of temporary blades. However, exposing the contralateral A1 may not 


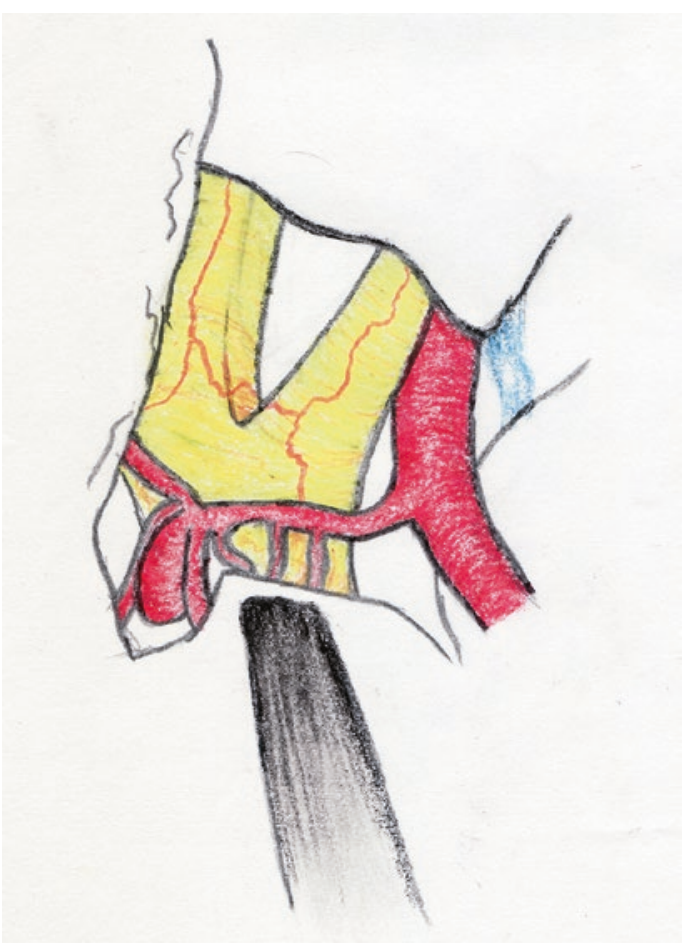

Fig. 14.7 After resecting the gyrus rectus, ipsi- and contralateral A2s should be dissected apart from the aneurysm neck to prepare it for the final clipping

be possible or safe in case of inferior or anteriorlooking aneurysms. In this step of surgery, the ICG-VA and endoscope are helpful to localize tiny perforating arteries which should be dissected off the aneurysm. The neck of the aneurysm should be dissected before the dome as it is likely to rupture. Any time during dissection if the chance of rupture is felt, insert proximal temporary clips.

There are some general precautions for clipping these aneurysms. The clip should be applied as parallel as possible to the parent artery. Tips of the clip should not be too long to injure the perforators/structures behind the aneurysm. To check for this, one can use endoscope, ICG-VA, or Doppler ultrasound.

\subsubsection{Superior Projecting Aneurysms}

The aneurysm neck might be attached to the proximal A2 bilaterally. So, enough space for the blades of the clip should be cleared on proximal
A2s and around the aneurysm neck. Before clipping, we usually check the contralateral proximal A2 with endoscope. Most AComA aneurysms can be obliterated with a straight aneurysm clip 1.5 times as much as the neck and parallel to the AComA. After clipping, contralateral A1-A2 complex and perforators should be checked with endoscope and ICG-VA.

\subsubsection{Anterior Projecting Aneurysms}

In these aneurysms, contralateral A1 and A1-A2 junction are usually obscured by the aneurysm. As dissection of the contralateral A1 is not possible before clipping the aneurysm, these aneurysms are usually approached from the side with dominant A1. A straight clip is usually applied parallel to the AComA to secure the aneurysm, and then, the patency of the contralateral A1 and A2 is checked with ICG-VA and endoscopy.

\subsubsection{Inferior Projecting Aneurysms}

The aneurysm dome is usually attached inferiorly to the optic chiasm, so, the frontal lobe should be retracted very gently to avoid premature rupture of these aneurysms. Again, as they cover the contralateral A1 artery, they should be approached from the side with dominant A1. A straight clip is usually needed to secure the neck. After clipping, the aneurysm should be dissected off the chiasma to release its pressure on optic system and check the patency of the contralateral A1 artery.

\subsubsection{Posterior Projecting Aneurysms}

As discussed earlier, these aneurysms are approached from the close A2 fork side. After dissecting behind the ipsilateral A2, the aneurysm is encountered with perforators stretched over its posterior wall. Hypothalamic artery that arises at the junction of A1-A2 is the most important perforator, though it is not present in all 
cases. Perforating arteries do not need to be entirely dissected off the aneurysm, and enough space for clip passage is all needed. A straight clip parallel to the AComA usually obliterates the aneurysm, but if the ipsilateral A2 is not mobilized anteriorly, a fenestrated clip to incorporate the A2 artery might be used. Again, check the integrity of the perforators and contralateral A2 at the conclusion of clipping.

\subsection{Surgeon Plan to Handle the Complication}

Immediate postoperative neurological deficits or incomplete recovery from anesthesia should be promptly worked up by a plain brain CT scan, and any major complication (e.g., surgical site hematoma) should be dealt with accordingly. As the normal brain CT scan does not rule out early cerebral ischemia, a vascular study and brain MRI might be indicated for further evaluation of the patient. Vasospasm after surgical treatment of ruptured aneurysms usually happens in distal anterior cerebral artery territories presenting with lower limb weakness. Though there is no strong evidence to support triple- $\mathrm{H}$ therapy, we keep the patient slightly hypertensive as soon as securing the aneurysm [5]. Nimodipine is administered prophylactically in all ruptured cases, and in case of symptomatic vasospasm, endovascular intra-arterial Papaverine is injected.

\subsection{Expert Opinion/Suggestion to Avoid Complication}

Venous infarction can happen due to prolonged brain retraction or coagulation of the veins during sylvian fissure dissection. Appropriate head and trunk positioning, wide sylvian fissure dissection with preserving the veins, extensive cisternal dissection and copious CSF drainage, and judicious gyrus rectus resection help the surgeon with exposing the aneurysm with minimal brain retraction. Intermittent release of the retractor should become a common practice to avoid venous drainage impairment and its consequences.
Optic apparatus injuries can happen due to direct trauma, compression from aneurysm clips, or arterial injuries. Meticulous cisternal dissection and preservation of tiny arterial feeders to the optic nerves should be performed to avoid such injuries. Also, both temporary and permanent clips (especially for inferior-looking aneurysms) should be placed in a trajectory that does not compress the visual structures.

Multimodality monitoring of the patients during aneurysm surgeries has been associated with improved outcome in recent years. Endoscopeassisted microsurgery of aneurysm is a common practice in many centers treating such pathologies. Visualizing the corners concealed behind the superficial structures gives some information about the contralateral A2 and perforating arteries and their relations to the hidden side of the aneurysm [4]. Also, after inserting the clip, the endoscopic view shows the relation of the clip blades to the perforators and confirms complete occlusion of the aneurysm neck.

Another monitoring modality is ICG-VA: it confirms appropriate occlusion of the aneurysm and the patency of the bilateral A2 and perforating arteries [3]. Though there are emerging data considering a role for FLOW 800 software of ICG-VA to quantify blood flow before and after clipping, we still do not rely on such semiquantitative data. Instead, whenever we want to measure blood flow and its changes before and after clipping (e.g., in aneurysms with severely atherosclerotic parent arteries), Doppler ultrasound (20 MHz probe) is used. Although recommended by some, we do not advocate routine intraoperative monitoring of somatosensory or motorevoked potentials for these patients.

\subsection{Things to Be Observed and Postoperative Care/ Follow-Up}

If the postoperative course is uneventful, we routinely perform 3D CT angiography and perfusion CT scan on the 7th postoperative day to check appropriate closure of the aneurysm neck and subclinical vasospasm on perfusion CT. However, if the patient develops neurological 
symptoms, an earlier CT angiography and brain MRI are performed, and if not conclusive, a digital subtraction angiography is requested. For patients presenting with $\mathrm{SAH}$, nimodipine will be continued for at least 2 weeks from the attack and tapered in case of normal perfusion CT scan.

\section{References}

1. Yamada Y, Kato Y, Ishihara K, Ito K, Kaito T, Nouri M, Ohede M, Inamasu J, Hirose Y. Role of endoscopy in multi-modality monitoring during aneurysm surgery: a single centre experience with 175 consecutive unruptured aneurysms. Asian J Neurosurg. 2015;10(1):52.
2. Kumar A, Kato Y, Motoharu H, Sifang C, Junpei O, Takeya W, Shuei I, Daikichi O, Yuichi H. An update on three-dimensional CT angiography in aneurysms: a useful modality for a neurosurgeon. Turk Neurosurg. 2013;23(3):304-11.

3. Oda J, Kato Y, Chen SF, Sodhiya P, Watabe T, Imizu S, Oguri D, Sano H, Hirose Y. Intraoperative nearinfrared indocyanine green-video angiography (ICG-VA) and graphic analysis of fluorescence intensity in cerebral aneurysm surgery. J Clin Neurosci. 2011;18(8):1097-100.

4. Kato Y, Sano H, Nagahisa S, Iwata S, Yoshida K, Yamamoto K, Kanno T. Endoscope-assisted microsurgery for cerebral aneurysms. Minim Invasive Neurosurg. 2000;43(2):91-7.

5. Dabus G, Nogueira RG. Current options for the management of aneurysmal subarachnoid hemorrhageinduced cerebral vasospasm: a comprehensive review of the literature. Intervent Neurol. 2013;2:30-51.

Open Access This chapter is licensed under the terms of the Creative Commons Attribution 4.0 International License (http://creativecommons.org/licenses/by/4.0/), which permits use, sharing, adaptation, distribution and reproduction in any medium or format, as long as you give appropriate credit to the original author(s) and the source, provide a link to the Creative Commons license and indicate if changes were made.

The images or other third party material in this chapter are included in the chapter's Creative Commons license, unless indicated otherwise in a credit line to the material. If material is not included in the chapter's Creative Commons license and your intended use is not permitted by statutory regulation or exceeds the permitted use, you will need to obtain permission directly from the copyright holder. 